\title{
Assessment of the rapid pullthrough technique in oesophageal manometry ${ }^{1}$
}

\author{
R J R GOODALL, D J HAY, AND J G TEMPLE ${ }^{2}$ \\ From the Department of Surgery, Hope Hospital (University of Manchester School of Medicine), Salford, \\ Lancashire
}

SUMMARY The lower oesophageal sphincter pressure was measured by infusion manometry using the rapid pullthrough technique in two studies designed to evaluate this technique. The first study performed was an assessment of its reproducibility in individual subjects on separate occasions. We conclude that the rapid pullthrough technique does not give reproducible results. The second study compared the lower oesophageal sphincter pressure obtained by this technique with that by the standard station pullthrough technique. We conclude that the results are not comparable.

The measurement of the pressure produced by the lower oesophageal sphincter (LOS) has been most commonly performed by the station pullthrough technique (SPT) using a multi-lumen, water infused catheter system. Although reservations about the reproducibility of this technique have been voiced (Perez-Avila and Irvin, 1975), we have recently confirmed that the reproducibility of the SPT is satisfactory (Hay et al., 1979). The SPT does, however, require cumbersome plumbing and the interpretation of the resultant recordings is frequently difficult because of the superimposition of respiratory and swallow waves. It may be impossible to obtain an analysable trace in an anxious subject.

The rapid pullthrough technique (RPT) described by Botha et al. (1957) abolished the respiratory excusions and swallow waves recorded by the SPT, thereby producing much simpler traces which are easier to interpret (Dodds et al., 1975).

The aims of this study were to assess the reproducibility of the RPT and to determine whether the values for lower oesophageal sphincter pressure measured by RPT were comparable with those obtained by SPT.

\section{Methods}

EQUIPMENT (Fig. 1)

The RPT was performed using a 6 FG ureteric catheter, $70 \mathrm{~cm}$ long and $2 \mathrm{~mm}$ in external diameter,

${ }^{1}$ Based on a communication read to the Surgical Research Society in July 1977.

2Address for reprints: Mr J G Temple, Queen Elizabeth Hospital, Queen Elizabeth Medical Centre, Edgbaston, Birmingham.

Received for publication 8 August 1979 as the recording probe. The most distal of the two side holes was sealed, leaving one orifice situated $1 \mathrm{~cm}$ from the tip of the catheter. The catheter was connected by thick-walled tubing to a Statham P23 series transducer. A further length of thickwalled tubing led to a greased glass syringe of $50 \mathrm{ml}$ capacity from which the catheter was infused with water at a rate of $1.8 \mathrm{ml} / \mathrm{min}$ using a Braun constant infusion pump. The pressures measured by the transducer were recorded on paper by a Grass multichannel recorder. With this system, occlusion of the recording orifice produced a pressure rise of $4.67 \mathrm{KPa} / \mathrm{s}(35 \mathrm{~mm} \mathrm{Hg} / \mathrm{s})$. All recordings were performed with the subject lying supine and withdrawal of the catheter was motorised at a constant rate of $2.4 \mathrm{~cm} / \mathrm{s}$. Fixed attachments of both perfusion tubing and withdrawal line ensured a reasonably constant radial orientation of the recording orifice.

While the catheter was being withdrawn through the lower oesophageal sphincter the subject suspended respiration alternatively at the end of normal inspiration and at the end of rormal exprration. Swallowing was also not permitted during catheter withdrawal. For each test the catheter was pulled through the !ower oeso phageal sphincter 10 times. The total duration of the test was thus about 10 minutes. To avoid discomfort resulting from the repeated manipulation of the catheter, a $25 \mathrm{~cm}$ oropharyngeal tube, through which the oesophageal probe passed, was placed into the proximal oesophagus.

In the second part of the study, where the SPT technique was also used, this was performed in the standard manner recently described (Hay et al., 1979) using a triple lumen polyvinyl catheter. Although only one withdrawal of the catheter was necessary 


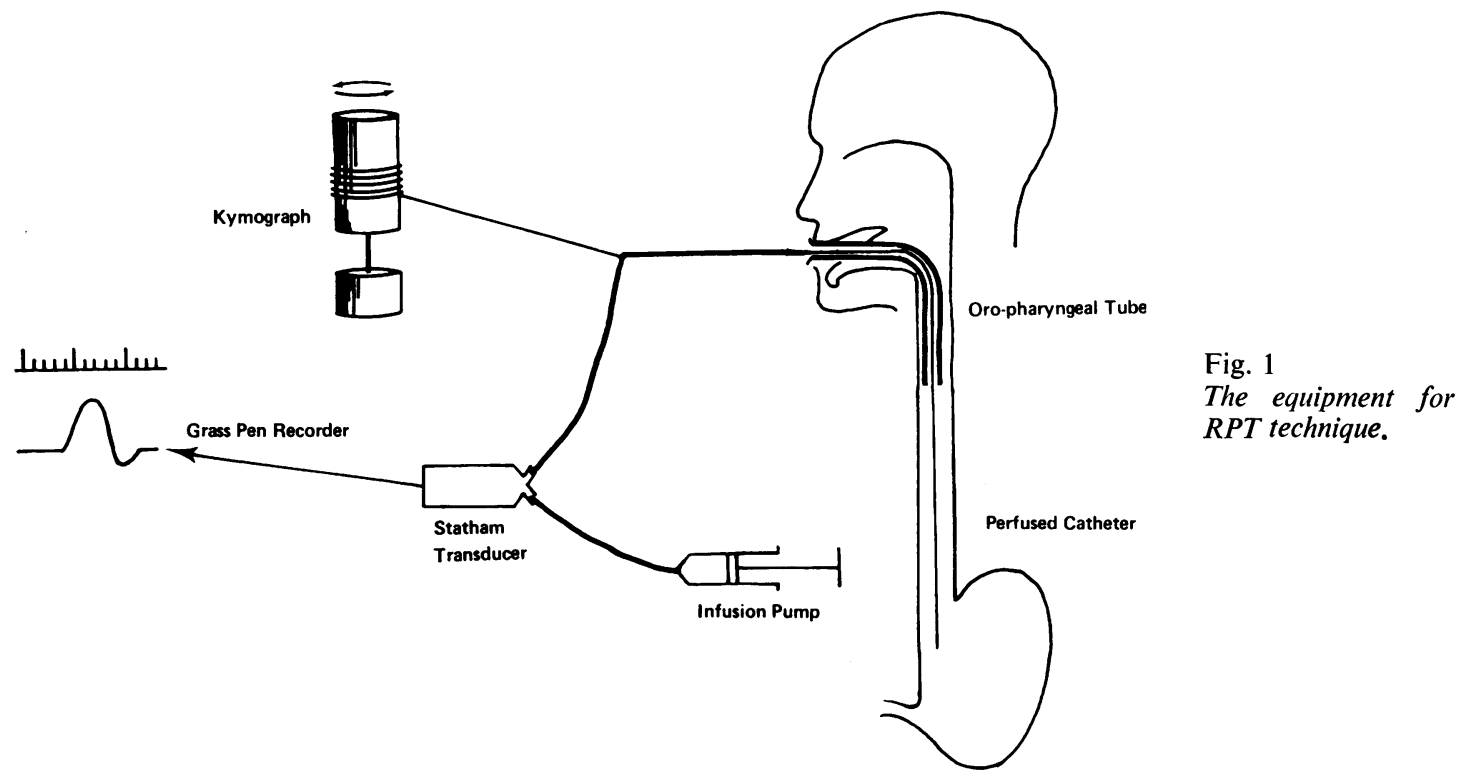

for the SPT technique, this test took between 30 and 45 minutes, as compared with the much shorter period for the RPT technique. Constant orientation upon introduction of this catheter was ensured by the inherent curvature of the assembly and thus reasonably constant orientation at the sphincter was achieved.

\section{SUBJECTS}

For the RPT reproducibility study 16 volunteers were used who were free from any symptoms of upper gastrointestinal disease. Informed consent was obtained in each case. The tests were performed after a six hour fast, during which time smoking was also forbidden. The RPT technique was performed on the same day at $8.30 \mathrm{a} . \mathrm{m}$. and again at 4.30 p.m. (day 1). The third test was performed at 8.30 a.m. one week later (day 7). (Two subjects did not have the test performed on the final occasion: one because of a sore throat and one was unable to swallow the tube).

For the second part of the study, 17 other volunteers were used; 10 were normal healthy subjects; of the remainder two were patients with non-stenosing duodenal ulceration, four were patients with proven gastro-oesophageal reflux, and one patient was studied three months after a successful Nissen fundoplication. As the intention of this part of the study was a comparison of techniques of RPT and SPT rather than the measurement of absolute values, the inclusion of patients with possibly abnormal sphincter pressure does not compromise the results. In these subjects, both SPT and RPT were performed consecutively but in random order.

\section{ANALYSIS OF TRACES (Fig. 2)}

Rapid pullthrough technique

For each recording the lower oesophageal sphincter a

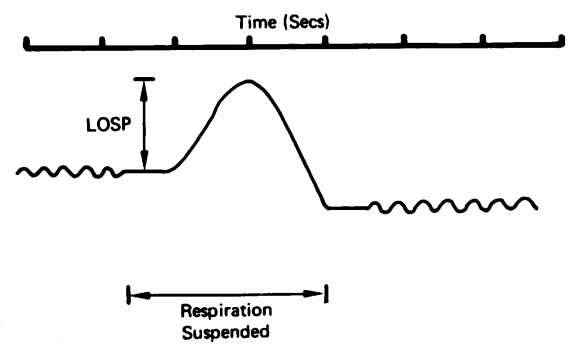

b

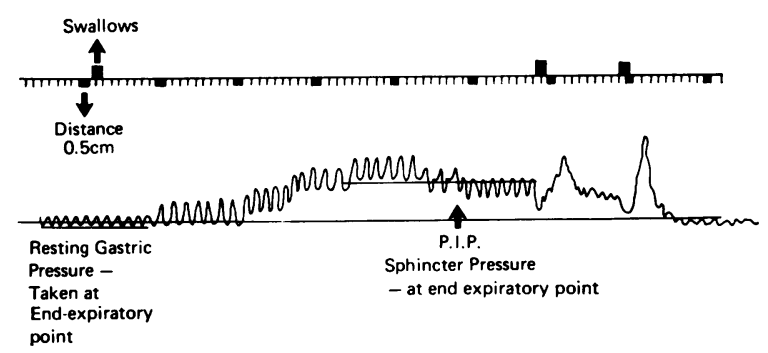

Fig. 2 Idealised traces (a) RPT and (b) SPT. 
pressure was taken as the difference between the mean intragastric pressure and the peak height of the trace. It has been expressed in kilopascals (with $\mathrm{mm}$ of mercury in parentheses). For each test in each subject, 10 values were obtained for LOS pressure. Analysis of the values showed that the correlation between the values recorded at the end inspiration and expiration was highly significant $(r=0 \cdot 82$, $\mathbf{P}<0.001)$ and similarly that there was significant correlation between values recorded at the beginning and end of each test $(r=0.74, \mathrm{P}<0.001)$. Therefore the LOS pressure was calculated as the mean of all 10 recorded values.

The length of the LOS was also determined. The time taken for the recording orifice to traverse the LOS was measured on the trace and the length derived from this as the speed of withdrawal was known and was constant.

\section{Station pullthrough technique}

The resting gastric pressure was taken as the mean of at least 10 consecutive end expiratory values and, in the same way, the pressure in the sphincter was taken as the mean of at least 10 consecutive end expiratory values around the pressure inversion point. The LOS pressure was therefore taken as the difference between the pressure in the sphincter and the intragastric pressure. For each individual three values were obtained for LOS pressure, one from each of the transducers, so therefore a mean LOS

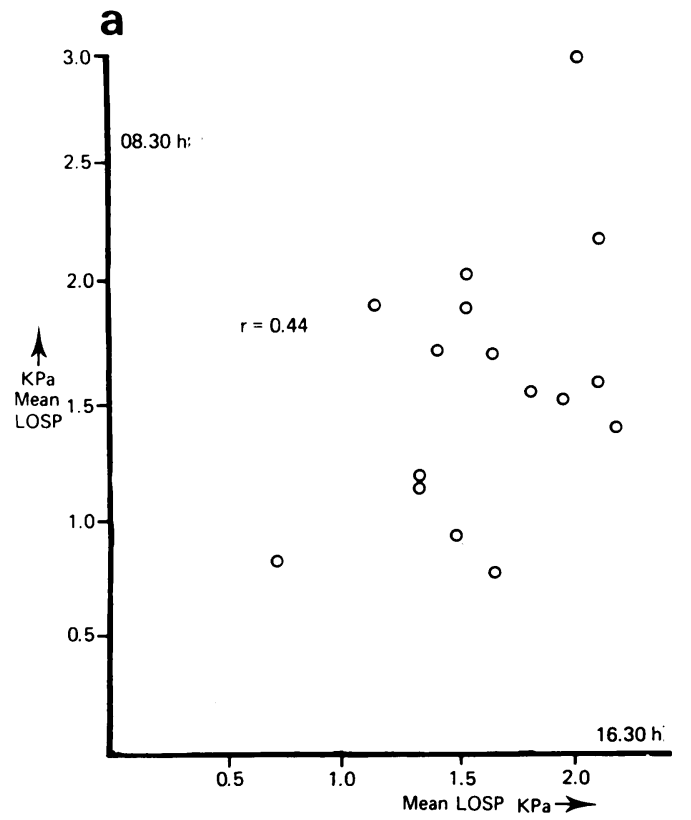

pressure was obtained by averaging these values and again this has been expressed in kilopascals (with millimetres of mercury in parentheses).

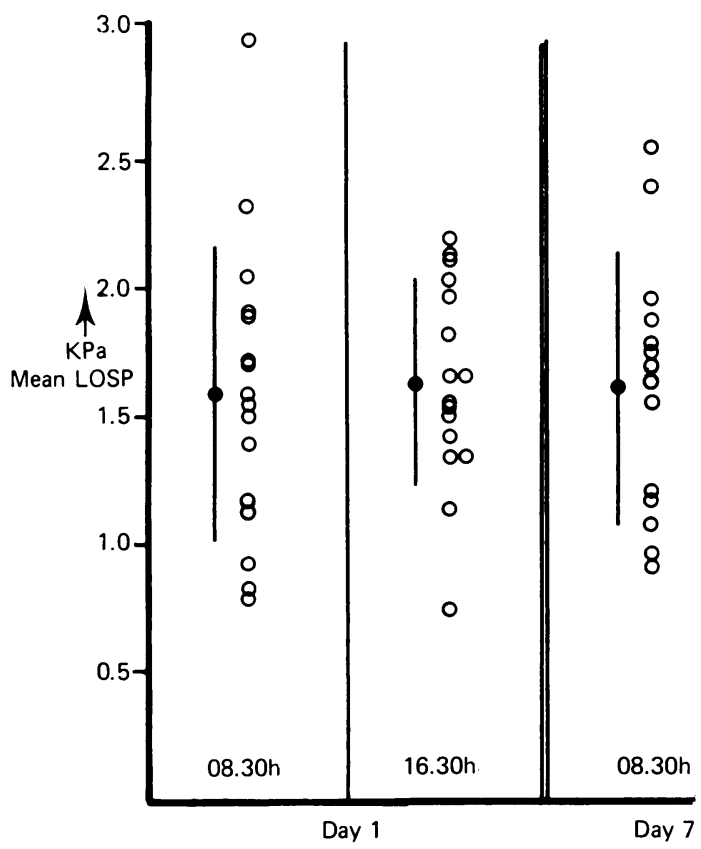

Fig. 3 Mean LOS pressure obtained by RPT on three separate occasions. Bars equal group mean $\pm S D$.

\section{b}

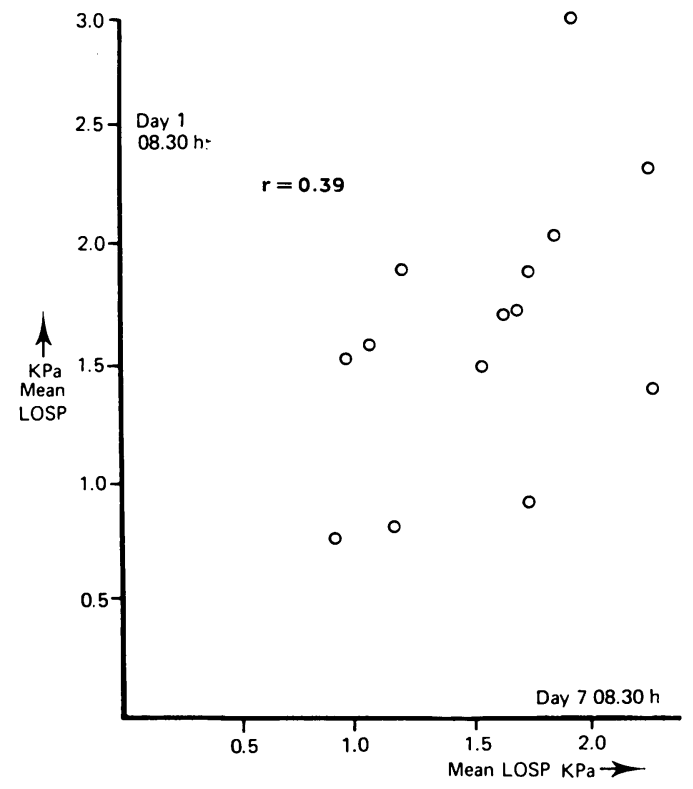

Fig. 4 Correlation of LOS pressure obtained by RPT (a) same day and (b) different days. 


\section{Statistical methods}

Paired Student's $t$ tests were used to compare the group mean values for each occasion in the first part of the study and for the two techniques in the second part.

Values for individuals either on the different occasion with RPT or from the different manometry techniques were compared both by the construction of correlation graphs and by one-way and two-way analysis of variance.

\section{Results}

\section{Reproducibility of $R P T$}

The results obtained for each subject on each of the three occasions are shown in Fig. 3. Two-way analysis of variance showed a significant difference between individuals $(P<0.005)$, but there was no statistical difference between the group mean values from occasion to occasion. The mean result we recorded for LOS pressure was $1.61 \mathrm{KPa} \pm \mathrm{SEM}$ $0.07(12.1 \mathrm{~mm} / \mathrm{Hg}$ SEM 0.6) (Fig. 4).

However, the values obtained on different occasions in any individual subject varied considerably and there was no correlation between results on the same day $(r=0.44)$ or on different days $(r=0 \cdot 43)$. One-way analysis of variance confirmed that variation within the observations on a single occasion was insufficient to account for the variation between occasions. Thus the technique is not reproducible in an individual subject.

The mean length of the LOS as measured by RPT was $4 \cdot 5 \mathrm{~cm} \pm$ SEM 0.11.

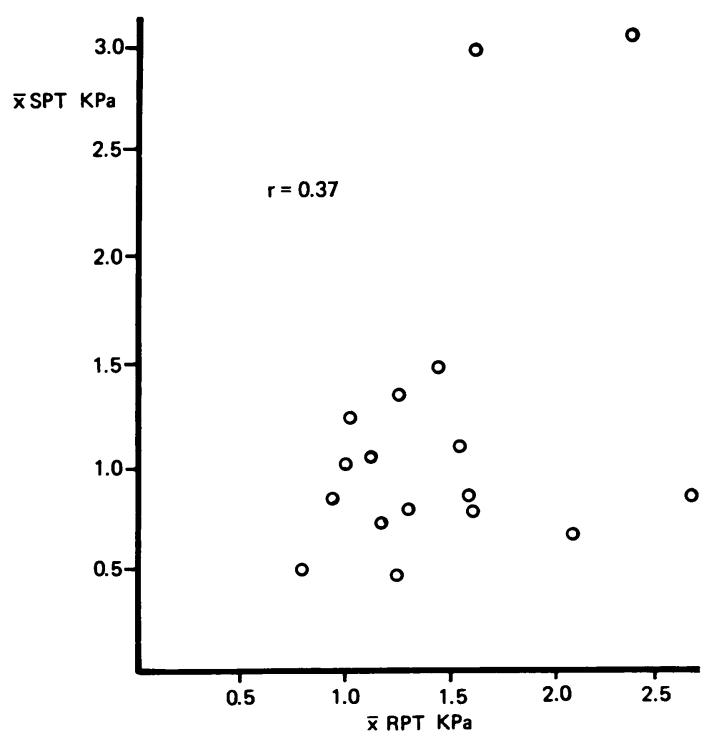

Fig. 5 Correlation of LOS pressure obtained by SPT and $R P T$.
Comparison of results obtained by $R P T$ and SPT (Fig. 5)

When the group mean values were compared using the two techniques they were very similar: RPT

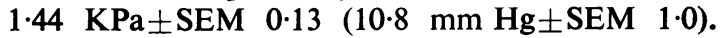
SPT $1.17 \mathrm{KPa} \pm$ SEM 0.19 (8.8 mm Hg \pm SEM 1.3), However, in individuals the correlation between the LOS pressure results obtained by RPT and SPT was poor $(r=0.37)$, and analysis of variance again showed this was not due to variation of observations in the individual tests.

It is of interest that the value for LOS pressure obtained by RPT in this group of volunteers was very similar to that in the first study from a different group.

\section{Discussion}

The results of our studies suggest that the RPT is not a reproducible technique. This is disappointing and contrary to the reported experience of Dodds et al. (1975). Our technique differed from that employed by Dodds $e t$ al. only in that the infusion rate used was less. We felt that at the high infusion rates they recommend the oesophagus would be flooded with fluid so that the pressures recorded in the LOS would not be resting pressures. Our lower infusion rate did produce an adequate pressure rise rate on occlusion, taking into account the average length of sphincter we expected and in fact observed.

The use of a recording catheter with only a single recording orifice has two disadvantages. First, no allowance can be made for the radial asymmetry known to occur in the LOS (Kaye and Showalter, 1971). With SPT the practice has been to record from several positions around the circumference of the LOS and then to take the mean of these values to give LOS pressure. In the technique of RPT that we employed a multi-lumen catheter was preciuded because of the need for the oropharyngeal tube: this ensured that the frequent manipulations of the catheter did not become intolerable for the patient. By ensuring that the orientation of the recording orifice was reasonably constant, we felt that any effect radial asymmetry might have upon the reproducibility of the technique would be minimal. The second disadvantage of a single lumen manometry catheter relates to its use in clinical situations. It is impossible from the resultant trace to identify any of the disorders of oesophageal motility which may mimic symptoms of gastro-oesophageal reflux. So for this reason its clinical usefulness may be limited.

The RPT technique has some favourable points. It is easier to perform than SPT and can easily be carried out by one operator. It is less time consuming 
and our subjects certainly found it more comfortable. This we attribute to the use of the oropharyngeal tube which made re-passage of the catheter after each pullthrough very simple. The traces obtained by the RPT technique are easier to interpret, being a simple waveform, than the SPT which frequently produces traces marred by swallowing, and respiratory fluctuations.

However, no matter how attractive its advantages, a system which cannot give reproducible results cannot be relied on to document the changes which may be caused by therapy. We have shown that the SPT technique, if performed with care and attention to detail, can give results which are reproducible both from hour to hour and day to day (Hay et al. 1979).

The lack of correlation between the results obtained for the station pullthrough technique and rapid pullthrough technique in the same subject in our study re-emphasises that differing recording systems always produce differing results for lower oesophageal sphincter pressure (Earlam, 1976; Hay et al., 1978).

\section{Conclusions}

Although the rapid pullthrough technique in lower oesophageal manometry possesses several attractive advantages over the station pullthrough technique the results obtained by this method must be viewed with caution at present until additional modification or adaptation of the technique can be shown to improve reproducibility to a satisfactory level.

The authors wish to thank Mr. M. K. Palmer, Chief Medical Statistician, Christie Hospital and Holt Radium Institute, Manchester, and Dr. R. J. M. Bennett for their advice and assistance with analysis of the results.

\section{References}

Botha, G. S. M., Astley, R., and Carre, I. J. (1957). A combined cineradiographic and manometric study of the gastro-oesophageal junction. Lancet, 1, 659-662.

Dodds, W. J., Hogan, W. J., Stef, J. J., Miller, W. N. Lydon, S. B. and Arndorfer, R. C. (1975). A rapid pullthrough technique for measuring lower esophageal sphincter pressure. Gastroenterology, 68, 437-443.

Earlam, R. (1976). Gastro-oesophageal junction. In Clinical Tests of Oesophageal Function, pp 1-40, chapter 1. Crossley Lockwood Staples: London.

Hay, D. J., Goodall, R. J. R., and Temple, J. G. (1979). The reproducibility of the station pullthrough technique for measuring lower oesophageal sphincter pressure. British Journal of Surgery, 66, 93-97.

Kaye, M. D., and Showalter, J. P. (1971). Manometric configuration of the lower esophageal sphincter in normal human subjects. Gastroenterology, 61, 213-223.

Perez-Avila, C., and Irvin, T. T. (1975). Interpretation of lower oesophageal pressure measurements (Abstract). British Journal of Surgery, 62, 663. 